\title{
Atuação do enfermeiro frente ao luto em tempos de pandemia
}

\author{
Nurse's role in grief in times of pandemic
}

El papel de la enfermera en el duelo en tiempos de pandemia

Rozemy Magda Vieira Gonçalves ${ }^{1 *}$, Terezinha de Fátima Gorreis ${ }^{1}$, Elisangela Souza ${ }^{1}$, Nicole Hertzog Rodrigues².

\section{RESUMO}

Objetivo: Relatar a vivência de enfermeiras que atuam em uma unidade de internação, frente à pacientes com múltiplas comorbidades diante do processo de morte e morrer em tempos de pandemia. Relato de experiência: Em meio às barreiras impostas pelo agravamento da pandemia, torna-se imperativo o apelo por suporte aos familiares enlutados por meio de uma escuta compreensiva e amparada, providenciando dados concretos sobre o desfecho do estado de saúde do paciente, elaborando e instituindo plano de cuidados de enfermagem singular a cada paciente/família. A instituição fornece suporte psicossocial para familiares acompanhantes em luto pré-perda, possibilitando espaço aberto para diálogo. Considerações finais: Percebe-se que alguns aspectos inseridos no processo de luto, seguem uma conjuntura sem precedentes na história recente e certamente, a terminalidade do processo de morrer pela COVID-19, se alarga em pessoas portadoras de doenças crônicas e com múltiplas comorbidades. Confronta-se com situações de dor e sofrimento, como nos cuidados de fim de vida, onde os relacionamentos interpessoais são ressignificados, seja com a família ou com a equipe assistencial.

Palavras-chave: Morte, Luto, Cuidados paliativos na terminalidade da vida.

\begin{abstract}
Objective: To report the experience of nurses working in an inpatient unit, facing patients with multiple comorbidities in the face of the process of death and dying in times of pandemic. Experience report: Amid the barriers imposed by the worsening of the pandemic, it is imperative to appeal for support to bereaved family members through a comprehensive and supported listening, providing concrete data on the outcome of the patient's health status, developing and instituting a care plan nursing unique to each patient/family. The institution provides psychosocial support for accompanying family members in pre-loss bereavement, providing an open space for dialogue. Final considerations: We realize that some aspects inserted in the grieving process follow an unprecedented situation in recent history and, certainly, the end of the process of dying by COVID-19, extends to people with chronic diseases and multiple comorbidities. We are faced with situations of pain and suffering, as in end-of-life care, we witness that interpersonal relationships are resignified, whether with the family or with the care team.
\end{abstract}

Key words: Death, Grief, Palliative care in the end of life.

\section{RESUMEN}

Objetivo: Informar la experiencia de enfermeras que laboran en una unidad de internación, enfrentando pacientes con múltiples comorbilidades ante el proceso de muerte y muriendo en tiempos de pandemia. Informe de experiencia: En medio de las barreras impuestas por el agravamiento de la pandemia, es

${ }^{1}$ Hospital de Clínicas de Porto Alegre (HCPA), Porto Alegre - RS. *E-mail: rozygong@gmail.com

2 Universidade Federal de Ciências da Saúde de Porto Alegre (UFCSPA), Porto Alegre - RS.

SUBMETIDO EM: 7/2021 
imperativo apelar al apoyo de los familiares en duelo a través de una escucha integral y acompañada, brindando datos concretos sobre el desenlace del estado de salud del paciente, desarrollando e instituyendo un plan de cuidados a cada paciente/familia. La institución brinda apoyo psicosocial a los familiares acompañantes en el duelo previo a la pérdida, brindando un espacio abierto para el diálogo. Consideraciones finales: Nos damos cuenta de que algunos aspectos insertados en el proceso de duelo siguen una situación inédita en la historia reciente y, ciertamente, el final del proceso de morir por COVID-19, se extiende a personas con enfermedades crónicas y múltiples comorbilidades. Nos enfrentamos a situaciones de dolor y sufrimiento, como en los cuidados al final de la vida, somos testigos de que las relaciones interpersonales se resignifican, ya sea con la familia o con el equipo de atención.

Palabras clave: Muerte, Duelo, Cuidados paliativos al final de la vida.

\section{INTRODUÇÃO}

O ambiente hospitalar acompanhado de sua tecnologia, é um espaço onde busca-se recuperar a saúde, tratar doenças e manter a vida. Porém, essa realidade não é uma constante, havendo momentos nos quais a morte ocorre fazendo parte do processo natural da vida (LEITE MR e MONTELO NMS, 2021). As Unidades de internação (UI) são espaços que viabilizam a manutenção do paciente sob assistência hospitalar por um período de 24 horas ou mais (COFEN, 2017).

O fenômeno da morte sofreu rupturas com o passar do tempo e cada cultura tem seus costumes relacionados a ela. Anteriormente, os eventos relacionados à morte aconteciam no ambiente doméstico, em um meio familiar onde o cerimonial da morte envolvia um corpo sem vida cercado de uma multidão de parentes e amigos. Na contemporaneidade, essa vivência é outra. Com o avanço da ciência e do saber médico, o corpo doente passou a ser hospitalizado e tratado, distanciando-o do convívio familiar e a morte passou a ser vista com maior estranheza e hesitação pela sociedade. Durante a pandemia de COVID-19, vimos acentuar-se esses fatores devido a tratar-se de um corpo doente e contagioso (ARIES P, 2017; SALUM MEG, et al., 2017).

A morte não deveria ser um acontecimento tão refutado diante de doenças progressivas e de difícil manejo. Pelo contrário, a morte deveria ser compreendida como um desfecho natural do agravo de muitas doenças e até mesmo como processo natural da vida. Mas a morte ainda é considerada um tabu e os profissionais de saúde sofrem abalos na saúde mental no enfrentamento de eventos que precedem a morte, muitas vezes não conseguindo a coparticipação junto a família, o que facilitaria a elaboração do processo de enlutamento (LEITE MR e MONTELO NMS, 2021).

E em meio à pandemia provocada pelo novo Coronavírus surgido em dezembro de 2019 em Wuhan na China e logo disseminado pelo mundo, mais uma vez nos defrontamos com a temida morte. A finitude da vida agora tem como agravante, os números rapidamente crescentes, aliados à ausência de um momento oportuno para despedidas dos entes queridos durante a internação ou permanência hospitalar (SIMPSON N, et al., 2020).

Com o surgimento da pandemia, foi possível observar que o mundo não estava preparado para lidar com a grandiosidade e especificidades da doença, com deficiências em relação às questões de planejamento e a preparação de equipes de saúde as quais se mostravam ineficientes (JUNIOR AMF, et al., 2020).

Embora tenha se passado mais de um ano de pandemia, ainda evidenciamos que, na hora da morte dos pacientes contaminados pela COVID-19, ainda há um vazio quanto a ausência do familiar e o sentimento de desespero destes ao serem vetados da despedida do seu ente querido. A discrepância entre o sentimento do enlutado e o reconhecimento de seu sofrimento por parte da comunidade em que está inserido pode reverberar de modo negativo na experiência do luto (OLIVEIRA DSA, et al., 2020).

A enfermagem é uma das profissões que atua na linha de frente e porque não dizer, mais próxima ao paciente nas áreas da saúde que atua cuidando do indivíduo que sofre (GONÇALVES JR e SIMÕES JRS, 2019). Busca assessorar tanto o paciente que está no final da vida, quanto o familiar que o acompanha 
presencialmente ou por chamadas de vídeo, criando arranjos que assegurem a todos, um atendimento holístico e humanizado, tanto quanto possível em meio aos desafios da pandemia da COVID 19 (MARTINS $A B$, et al., 2020).

Portanto, em detrimento do número elevado e crescente de óbitos hospitalares no contexto de pandemia da COVID-19, o objetivo deste estudo é relatar a vivência de enfermeiros que atuam em uma unidade de internação, que atende pacientes com múltiplas comorbidades frente ao processo de morte e morrer em tempos de pandemia.

\section{RELATO DE EXPERIÊNCIA}

Trata-se de um estudo descritivo, tipo relato de experiência sobre a assistência de enfermagem ao paciente e familiares frente ao processo de morte e morrer durante a pandemia da COVID-19 em um hospital universitário do sul do país. O campo de estudo foi uma unidade de internação clínica de um hospital universitário no sul do país que atende majoritariamente a pacientes do Sistema Único de Saúde (SUS). Este hospital, além de ser referência no atendimento, ensino e pesquisa de inúmeras especialidades, tornou-se referência também no atendimento à população frente ao advento da COVID-19. A unidade referida atende diversas especialidades em relação a doenças clínicas especialmente, as doenças de etiologia cardíaca, vasculares cerebrais e pneumológicas, sendo muitas dessas condições consideradas terminais.

Durante o período de pandemia, o hospital adotou como uma das medidas preventivas para o controle da disseminação da COVID-19, a restrição da circulação de pessoas na instituição. Às visitas diárias foram suspensas e os acompanhantes, passaram a ser solicitados apenas em casos especiais a exemplo, para pacientes instáveis, com riscos de quedas, fuga ou sangramentos ou muito dependentes (que apresentam condições incapacitantes, determinadas condições mentais, físicas ou patológicas que afetam a funcionalidade, acometendo geralmente indivíduos com múltiplas comorbidades).

Em relação ao risco de quedas, a medida preventiva utilizada para liberação de acompanhante, tem sido de pacientes com risco de queda elevado conforme pontuação na escala Severo-Almeida-Kuchenbecker (SAK) (SEVERO IM, 2015), que é uma ferramenta atualizada de grande valia, que ampara o enfermeiro na classificação de risco e aplicação das intervenções preventivas para o evento, a partir do diagnóstico de enfermagem "Risco de Quedas". A aplicação da sistematização da assistência de enfermagem utilizando-se de ferramentas como o processo de enfermagem e escalas de classificação, impactam diretamente na segurança do paciente. Estes achados são compatíveis com os indicadores institucionais que demonstram baixas taxas de quedas em pacientes hospitalizados (LUIZA MF, et al., 2014).

Em meio essa medida de contenção da circulação imposta pelo agravamento da pandemia, e em meio às mortes inerentes ao ambiente hospitalar, buscou-se apoiar os familiares enlutados através de uma escuta empática e acolhedora, fornecendo informações precisas sobre o prognóstico do paciente, elaboração do plano de cuidados de enfermagem caso a caso.

A instituição, tendo em vista que o ideal no processo de adoecimento e morte, seria de possibilitar a presença do acompanhante, passou a fornecer suporte psicossocial para familiares acompanhantes e aos familiares em luto pré-perda, criando formas de contornar obstáculos, principalmente através do uso da tecnologia com chamadas de vídeo e ligações telefônicas.

Quando a família não pode estar presente, a equipe multidisciplinar que assiste o paciente na atenção hospitalar, buscou acolher todas as necessidades existentes olhando integralmente cada contexto de forma holística e humanizada dentro de suas peculiaridades e buscando superar obstáculos presentes na conjuntura atual buscando diferentes alternativas quando a presença do familiar não se fazia possível.

Como as visitas presenciais foram suspensas, optou-se por repassar informações sobre o quadro clínico do doente via telefone, conduta reconhecida como prudente, mas que se apresenta como fonte de maior angústia e sofrimento para os familiares. Assim, passou-se a liberar apenas um familiar para acompanhar este processo de terminalidade restringindo a despedida podendo tanto sobrecarregar o familiar como entristecer o doente terminal. 
Situação agravada quando o paciente falece, pois, os rituais de despedida também sofreram alterações, inclusive, as instituições sanitárias determinaram que os velórios passassem a ser mais curtos, o caixão obrigatoriamente deve se manter lacrado. Além disso, em algumas situações, a família espera alguns dias para que o enterro possa acontecer, pois o fluxo de sepultamentos tornara-se intenso em muitos cemitérios (OLIVEIRA DSA, et al., 2020).

Outro agravante é que, durante a atual pandemia da COVID-19, provavelmente as pessoas não planejaram seus cuidados de finitude de vida, não discutiram previamente com a família seus desejos e preferências, caso fiquem muito debilitados e venham a morrer (MOORE KJ, et al., 2020). Isso pode gerar conflitos de desejos entre familiares sobre o que é mais adequado para seu ente querido neste momento doloroso e intempestivo.

Acompanhamos diariamente pacientes que apresentam prognóstico desfavorável, com restrição de investimento ou em cuidados paliativos. Estas situações são notoriamente complicadas, à medida em que as famílias precisam tomar decisões difíceis em relação à aplicação ou não de ressuscitação cardiorespiratória, ou em relação ao tratamento a ser aplicado em seu ente querido inclusive, nos pós morte (MOORE KJ, et al., 2020).

Como a instituição é referência no atendimento de casos suspeitos e confirmados de COVID-19, acaba recebendo pacientes procedentes de todo estado do Rio Grande do Sul e alguns casos de outras regiões do Brasil. Por vezes, essa distância também se torna um impeditivo a presença de familiares durante a internação hospitalar.

A instituição optou por contratação emergencial de funcionários para suprir a demanda de recursos humanos durante a pandemia da COVID-19. Isso surge como um facilitador já que pesquisa demonstra que o número insuficiente de profissionais é um dos fatores que dificulta a possibilidade de um último contato, entre os pacientes internados e seus familiares sendo esse, considerado um cuidado muito importante (OLIVEIRA DSA, et al., 2020).

Frente ao contexto apresentado, verificou-se o esforço da equipe assistencial de enfermagem para a promoção do conforto aos familiares frente a morte e morrer dos seus entes queridos num momento atípico e de enfrentado mundial de uma pandemia. Sabe-se que esse momento exacerba vários sentimentos como raiva, impotência, hostilidade entre outros. Mas a enfermagem que está na linha de frente nessa situação tem de procurar manter o equilíbrio emocional, o respeito e a empatia para a promoção do cuidado humanizado (SALUM MEG, et al., 2017).

\section{DISCUSSÃO}

Em dezembro de 2019, o betacoronavírus SARS-CoV-2 foi identificado pela primeira vez em humanos em Wuhan, China. Um surto local inicial de infecção respiratória culminou em uma pandemia global (COVID-19), como o declarado pela Organização Mundial da Saúde. Devido ao rápido crescimento dos casos da COVID19, muitos países ativaram planos de emergência e desenvolveram diretrizes para controlar a doença e defender a saúde pública por meio de intervenções de distanciamento social, como suspender atividades escolares, restrições de viagens, restrição de visitas hospitalares e confinamento em casa (SANTOS CF, 2020).

Os enfermeiros que trabalham na chamada "linha de frente" (em contato direto com os infectados), tem buscado dar apoio emocional e manter a humanização do cuidado no final da vida, mesmo em meio a tantas outras tarefas relacionadas ao cuidado físico das pessoas hospitalizadas com COVID-19 (INGRAVALLO F, 2020). Mesmo diante das contratações emergenciais ocorridas, nos deparamos com profissionais com habilidades e conhecimentos que ainda precisam ser aprimorados. Dada à escassez de profissionais especialistas em saúde mental e a sobrecarga dos serviços que se mostra inapta para realizar o tratamento das pessoas necessitadas durante o período da pandemia (VASCONCELOS SE, et al., 2020).

A caracterização dos rituais e do processo de luto está intimamente relacionada ao modo como as pessoas resolvem as questões relativas ao próprio desenrolar da vida social do qual a morte faz parte (SOUZA CP e 
SOUZA AM, 2019). No contexto da pandemia a qual vivenciamos, o processo de morte, morrer e elaboração do luto foi alterado, muito devido às restrições relacionadas aos rituais de despedida, funerais e enterros. Tal conjuntura pode alterar o processo de luto e assimilação da morte tornando-a uma experiência dolorosa e problemática para a família e comunidade (EISMA MC, et al., 2020; FUNDAÇÃO OSWALDO CRUZ, 2020).

O paciente acometido pela COVID-19 passa por momentos delicados, como inconsciência e entubação que podem durar dias e, no caso de melhora, são seguidos por dias de confusão, desorientação espaçotemporal, polineuropatias, alimentação prejudicada, utilização de dispositivos médico-hospitalares acrescidos do sofrimento de não ter próximo fisicamente seus familiares e amigos, a rede de apoio que lhe ofertaria segurança e bem-estar (OLIVEIRA DSA, et al., 2020).

Nesses casos em que os familiares não conseguem ter contato direto com os pacientes que estão isolados em enfermarias próprias de quem contraiu a COVID-19 ou pós contágio, o espaço para a fala, ressignificações, reavaliações, e despedidas tende a ser limitado ou ainda, podem não ocorrer. Essas limitações têm sido enfrentadas por meio de ligações e chamadas de vídeo, além, da transmissão de mensagens repassadas dos familiares pelos profissionais de saúde que assistem o paciente integralmente (OLIVEIRA DSA, et al., 2020).

A prática do isolamento social e da quarentena são essenciais para o controle da propagação dessa doença infectocontagiosa, porém a preocupação com a saúde em geral não deve ser negligenciada (REIS MAOM, et al., 2021). Percebemos que a pandemia da COVID-19 demonstrou, na prática, que mesmo com o avanço tecnológico, a humanidade continua frágil diante do desconhecido (SOUZA-JUNIOR JR, et al., 2020).

Percebeu-se que algumas particularidades entrelaçadas no processo de luto, seguem uma conjuntura sem precedentes na história recente e certamente, a terminalidade do processo de morrer pela COVID-19, se alarga em pessoas portadoras de doenças crônicas e com múltiplas comorbidades (DANTAS CR, et al., 2020).

Diariamente nos confrontamos com situações de dor e sofrimento, como nos cuidados no fim da vida. Presenciamos que os relacionamentos interpessoais são ressignificados, seja com a família ou com a equipe assistencial na atenção hospitalar. Através deste estudo percebemos que a atuação multiprofissional ascendeu saberes e instigou conhecimentos dos profissionais implicados no transcurso do luto na atenção terciária após fenômeno coletivo como a pandemia. E ainda, tais condições exigem um ágil incremento de intervenções para a promoção do cuidado no zelo ao luto durante a pandemia da COVID-19. Essa condição de luto pode ser dita como um fato novo e a necessidades de outros estudos se faz presente.

\section{REFERÊNCIAS}

1. ARIES P. História da morte no Ocidente: da idade média aos nossos dias. Rio de Janeiro: Nova Fronteira, 2017.

2. CONSELHO FEDERAL DE ENFERMAGEM (COFEN). RESOLUÇÃO COFEN-543 de 12 mai 2017. Dispõe sobre os parâmetros mínimos para dimensionar o quantitativo de profissionais das diferentes categorias de enfermagem para os serviços/locais em que são realizadas atividades de enfermagem. Brasil, 2017. Disponível em: http://www.cofen.gov.br/resolucao-cofen-5432017_51440.html.

3. DANTAS CR, et al. O luto nos tempos da COVID-19: desafios do cuidado durante a pandemia. Rev. Latino am. Psicopat. Fund., São Paulo, 23(3), 509-533, set. 2020.

4. EISMA MC, et al. Prolonged grief disorder following the Coronavirus (COVID-19) pandemic. Psychiatry Res, 2020; 288: 113031.

5. FUNDAÇÃO OSWALDO CRUZ (FIOCRUZ). Saúde mental e atenção psicossocial na pandemia COVID-19: processo de luto no contexto da COVID-19. Rio de Janeiro. 2020. Disponível em: https://www.fiocruzbrasilia.fiocruz.br/wpcontent/uploads/2020/04/sa\%c3\%bade-mental-e-aten\%c3\%a7\%c3\%a3o-psicossocial-na-pandemia-covid-19processo-de-luto-no-contexto-da-covid-19.pdf. Acesso em 18: de jun. 2021.

6. GONÇALVES JR, SIMÕES JRS. A percepção do enfermeiro no lidar com a morte durante a assistência. Revista JRG de Estudos Acadêmicos, 2019; 2(5): 166-182.

7. INGRAVALLO F. Death in the era of the COVID-19 pandemic. The Lancet Public Health, 2020; 5(5): e258.

8. JUNIOR AMF, et al. COVID-19 em profissionais da saúde, vivências e perspectivas: um relato de experiência. Revista Acervo Saúde, 2020; 12(12): 1-5.

9. LEITE MR, MONTELO NMS. Profissionais de saúde e sua relação com a morte e o morrer de pacientes em UTI. Revista Acervo Saúde, 2021; 13(2): 1-8. 
10. LUIZA MF, et al. Mapeamento de cuidados de enfermagem para pacientes com risco de quedas na Nursing Interventions Classification. Rev Esc Enferm USP, 2014; 48(4):632-9.

11. MARTINS AB, et al. A assistência multiprofissional a pacientes em tratamento de COVID-19 e a minimização do distanciamento familiar em um serviço de pronto atendimento em Manaus, Amazonas. Revista Eletrônica Acervo Saúde, 2020; 12(12): 1-6.

12. MOORE KJ, et al. Supporting families in end-of-life care and bereavement in the COVID-19 Era. Int Psychogeriatr, 2020;32(10):1245-1248.

13. OLIVEIRA DSA, et al. Processo de luto diante da pandemia: repercussões frente à Covid-19 no Brasil. Revista KairósGerontologia, 2020; 23(28): 499-216.

14. REIS MAOM, et al. Impactos na saúde mental por distanciamento e isolamento sociais pela COVID-19: uma perspectiva brasileira e mundial. Revista Eletrônica Acervo Saúde, 2021; 13(2): 1-7.

15. SALUM MEG, et al. Processo de morte e morrer: desafios no cuidado de enfermagem ao paciente e família. Revista Rene, 2017; 18(4):528-35.

16. SANTOS CF. Reflections about the impact of the SARS-COV-2/COVID-19 pandemic on mental health. Braz J Psychiatry, 2020; 42(3): 329-332.

17. SEVERO IM. Modelo de predição de risco de quedas em pacientes adultos hospitalizados: derivação e validação de um escore. Tese (Doutorado em Enfermagem) - Programa de Pós-Graduação em Enfermagem. Universidade Federal do Rio Grande do Sul, Porto Alegre, 2015; 141 p.

18. SIMPSON N, et al. Don't forget shared decision-making in the COVID-19 crisis. Internal Medicine Journal, 2020; 50(6): 761-763.

19. SOUZA CP, SOUZA AM. Rituais Fúnebres no Processo do Luto: Significados e Funções. Psicologia: Teoria e Pesquisa, 2019; 35: e35412.

20. SOUZA-JUNIOR JR, et al. COVID-19 e a promoção da saúde em tempos de pandemia. Revista Eletrônica Acervo Saúde, 2020; 46(46): 1-7.

21. VASCONCELOS SE, et al. Impactos de uma pandemia na saúde mental: analisando o efeito causado pelo COVID19. 2020. Revista Eletrônica Acervo Saúde, 2020; 12(12): 1-7. 